\title{
Clinical Semiology and Neuroradiologic Correlates of Acute Hypernatremic Osmotic Challenge in Adults: A Literature Review
}

\author{
F.Y. Ismail, A. Szóllics, M. Szólics, N. Nagelkerke, and M. Ljubisavljevic
}

\begin{abstract}
SUMMARY: The complex interplay between hypernatremic osmotic disturbances and cerebral lesions is yet to be clarified. In this review, we discuss, on the basis of the reported data of hypernatremic CNS challenge in the adult population, the clinical and radiologic features of the condition. Our search captured 20 case studies and 1 case series with 30 patients in total who acquired acute hypernatremia due to different etiologies and developed CNS lesions. We explored the associations between premorbid conditions, clinical presentation, hypernatremic state, correction rate, and radiologic appearance, including the localization of brain lesions and the outcomes. The results revealed that altered mental status was the most commonly reported symptom and osmotic demyelination syndrome in the form of extrapontine myelinolysis was the prevailing radiologic pattern. Finally, we contrasted, when appropriate, clinical and experimental data related to hypernatremic and hyponatremic osmotic insults to aid the understanding of the pathophysiology of CNS osmotic brain injury.
\end{abstract}

ABBREVIATIONS: $B G$ = basal ganglia; $C P M=$ central pontine myelinolysis; EPM = extrapontine myelinolysis; GCS = Glasgow Coma Scale; ODS = osmotic demyelination syndrome

$\mathrm{T}$ he associations between hyponatremic osmotic disturbances and cerebral lesions are well-established and studied. Classically, the lesions were recognized to involve demyelination in the pons. ${ }^{1}$ The term "central pontine myelinolysis" was devised to delineate the character of pathologic insult. Subsequently, the concept was extended with the recognition that lesions may occur beyond the pons. Thus, the term "extrapontine myelinolysis" was coined. Although initially recognized to appear in the malnourished and alcoholics, ${ }^{1} \mathrm{CPM} / \mathrm{EPM}$ has also been reported in adults with a variety of serious illnesses, and a link between these disorders and the rapid correction of sodium in patients with hyponatremia was eventually established. Finally, an umbrella "osmotic demyelination syndrome" term, which included both CPM and EPM, was coined. CPM and EPM are believed to be the same disease, sharing the same pathology, associations, and time course but differing in clinical manifestations. However, it has been in-

From the Departments of Pediatrics (F.Y.I.) and Physiology (M.L.) and Institute of Public Health (N.N.), College of Medicine and Health Sciences, United Arab Emir ates (UAE) University, Al Ain, UAE; Department of Radiology (A.S.), Kálmán Pándy Békes County Hospital, Gyula, Hungary; and Division of Neurology (M.S.), Department of Medicine, Tawam Hospital, Al Ain, Abu-Dhabi, UAE.

Please address correspondence to M. Ljubisavljevic, MD, PhD, Department of Physiology, Faculty of Medicine and Health Sciences, UAE University, PO Box 17666, Al Ain, United Arab Emirates; e-mail: milos@uaeu.ac.ae

- Indicates open access to non-subscribers at www.ajnr.org

三 Indicates article with supplemental on-line table.

http://dx.doi.org/10.3174/ajnr.A3392 creasingly recognized that ODS can occur in the setting of other osmotic challenges such as hyperglycemia ${ }^{2-5}$ and hypoglycemia, ${ }^{6,7}$ as well as normonatremia ${ }^{8}$ and hypernatremia. ${ }^{9}$

Furthermore, experimental data suggest that different anatomic locations in the brain may have different susceptibility to osmotic stress, depending on their ionic, organic, and inorganic makeup. These subtle variations of the CNS osmotic "ecosystem" may influence the response and effectiveness of processes of adaptation (during insult) and de-adaptation (during recovery) of the brain. ${ }^{10}$

While hyponatremic insult has been widely examined and reported, the hypernatremic osmotic insult, primarily due to its comparative scarcity in adults, is relatively poorly described and understood. Therefore, it is still unclear whether the vulnerability of different brain regions to osmotic stress is similar in states of hyponatremia and hypernatremia and whether they share similar clinical and radiologic presentations.

\section{OBJECTIVES}

In this review, we discuss the clinical and neuroradiologic presentations of acute hypernatremic CNS injury in the adult population on the basis of the reported literature data. We also explore the associations between premorbid conditions, clinical presentation, hypernatremic state, correction, location of brain lesions, and clinical outcomes. Finally we compare, when appropriate, clinical and experimental data in the setting of hypo- and hyper- 
natremia with the aim of better understanding the pathophysiology of CNS osmotic brain injury.

\section{METHODOLOGY}

We conducted PubMed and ScienceDirect database searches (until June 3, 2012) by using the following keywords: hypernatremia AND demyelination, hypernatremia AND myelinolysis, hypernatremia AND CNS, hypernatremia AND cortical laminar necrosis, hypernatremia AND edema, hypernatremia AND MR imaging, hypernatremia AND imaging, hypernatremia AND CT, and hypernatremia AND brain. This initial strategy yielded 697 articles in PubMed and 242 articles in ScienceDirect, with significant overlap. English and non-English articles were initially populated, and reference lists in retrieved reports were examined. Five nonEnglish articles were not included (Japanese, Danish, French, and 2 in Spanish) because the original manuscripts were not available.

We included and further analyzed studies reporting clinical and radiologic cases that fulfilled the following criteria: patients older than 18 years of age, acute hypernatremia (sodium $>145$ $\mathrm{mEql} / \mathrm{L}$ ) developing within 48 hours, presence of abnormal neurologic signs and symptoms, and documented neuroradiologic investigation. Exclusion criteria included the following: presence of a hyponatremic state (sodium $<135 \mathrm{mEql} / \mathrm{L}$ ) before the onset of symptoms and presence of CNS pathology that might account for or mask abnormal radiologic findings. When available, initial and highest sodium ion $(\mathrm{Na}+)$ levels and the rate and duration of the correction were also examined.

Descriptive analysis, linear regression, 2-sided Student $t$ test, Mann-Whitney and Fisher exact tests, linear-by-linear association, and binary logistics were used to analyze categoric and continuous data. Statistical significance was defined as $P<.05$. To further explore the associations among radiologic features, we developed a demyelination score (0-6) that represents the sum of the number of areas affected by demyelination: pons, white matter, corpus callosum, basal ganglia, cerebellum, and cortex.

\section{RESULTS}

A total of 20 case studies and 1 case series in English, Spanish, and Japanese languages reporting 30 adult patients who developed acute hypernatremia due to different etiologies with abnormal clinical and neuroradiologic findings (Table) were eventually included.

\section{Clinical Characteristics}

The age of patients ranged between 18 and 73 years (mean age, $36.93 \pm 16.9$ years) (On-line Table). Twenty-four (80\%) were women.

The patients were clustered on the basis of morbid history into 4 groups: previously healthy individuals, patients with direct CNS comorbidity, patients with indirect CNS comorbidity, and patients with non-CNS comorbidities. "Direct nervous system comorbidity" denotes the presence of CNS pathology (eg, tumor or hydrocephalus), while "indirect CNS comorbidity" represents conditions that have secondary pathologic effects on the CNS (eg, liver failure and/or hepatic encephalopathy). The rationale behind such clustering was to highlight conditions that might mask the development of abnormal neuroradiologic findings and/or render the brain more vulnerable to osmotic challenges.

Thirteen patients $(43.3 \%)$ were previously healthy with no pre- or comorbid conditions. Ten patients (33.3\%) had non-CNS comorbidities that were not etiologically related to acute hypernatremia per se, including hydatid cyst of the liver $(n=5)$, heart disease $(n=1)$, gestational diabetes mellitus $(n=1)$, acute respiratory distress syndrome $(n=1)$, chronic lithium therapy ( $n=$ 1 ), and chronic alcoholism $(n=1)$. Three patients had direct CNS comorbidities $(10 \%)$ in the form of suprasellar tumor $(n=1)$, lymphocytic neuroinfundibulohypophysitis $(n=1)$, and pituitary craniopharyngioma and hydrocephalus $(n=1)$, while 4 patients $(13.3 \%)$ had indirect CNS comorbidity, including endstage liver disease and renal failure.

The etiologies of acute hypernatremia in the reported cases were categorized from a pathophysiologic point of view into 2 main categories: exogenous sodium overload in $36.7 \%(n=11)$, and hypotonic volume depletion in $63.3 \%(n=19)$ of patients. In cases of exogenous sodium overload, we identified the following factors: post-liver transplantation $(n=1)$, post-heart surgery $(n=1)$, hydatid cyst lavage with hypertonic saline $(n=5)$, excessive lactulose $(n=1)$, dialysis error $(n=1)$, sodium bicarbonate therapy for end-stage liver disease $(n=1)$, and nonaccidental sodium chloride ingestion $(n=1)$. The following predisposing factors were reported in cases of volume depletion: central diabetes insipidus $(n=5)$, nephrogenic diabetes insipidus $(n=1)$, postpartum dehydration $(n=11)$, hunger strike $(n=1)$, and anorexia $(n=1)$.

The presenting symptoms, reported in decreasing frequency, were the following: altered mental status ranging from apathy, inattention, and confusion to deep coma in $76.7 \%(n=23)$; motor deficit mainly in the form of quadriparesis/plegia in $66.7 \%$ $(n=20)$; myoclonic or secondary generalized tonic clonic seizures in $43.3 \%(n=13)$; and cranial nerve dysfunction, including bulbar symptoms such as dysphagia and dysarthria, in $43.3 \%$ ( $n=$ 13 ) of patients. Glasgow Coma Scale scores on presentation ranged from 3 to 14 (mean, 8.6 \pm 3.2 ). While the exact GCS score was reported in 4 studies, in other studies the reported clinical presentation was used to estimate the GCS score (13 studies). In 3 studies, the provided clinical description did not allow estimating GCS.

The information about the presence and extent of concomitant electrolyte abnormalities was incomplete. Therefore, we limited our comparison to changes in initial $\mathrm{Na}^{+}$and osmolality, which were reported in all studies. The initial $\mathrm{Na}^{+}$level at the time of presentation was $142-200 \mathrm{mEq} / \mathrm{L}$ (mean, $175.1 \pm 16.8 \mathrm{mEq} / \mathrm{L}$ ). Serum osmolality ranged between 314 and $459 \mathrm{mOsm} / \mathrm{kg}^{-1}$ (mean, $387.6 \pm 39.9 \mathrm{mOsm} / \mathrm{kg}^{-1}$ ).

Data for 18 subjects were not reported in detail, while in those reported, the correction rate was variable. The duration of correction was as rapid as 13 hours and as slow as 5 days. More specifically, $\mathrm{Na}^{+}$ was corrected within 24 hours in 4 patients (33.3\%), within 48 hours in 3 patients (25\%), and in $>48$ hours in 5 patients $(41.7 \%)$.

\section{Neuroradiologic Findings}

All patients underwent neuroimaging ( $\mathrm{CT}$ and/or MR imaging) at certain points in the course of their illnesses. Neuroradiologic 
findings were grouped into 4 patterns that are not mutually exclusive: central pontine myelinolysis, extrapontine myelinolysis, vascular changes, and cerebral and CSF volume changes. In 2 patients, the neuroimaging findings were normal $(6.7 \%) .{ }^{11,12}$

\section{Osmotic Demyelination Syndrome}

ODS was found in $80 \%$ of patients $(n=24)$. CPM was evident as an isolated pathology in $16.7 \%$ of patients $(n=5)$. Pontine demyelination was located either centrally or dorsolaterally with hyperintense T2 signal.

On the other hand, "stand-alone" EPM was demonstrated in $33.3 \%$ of patients $(n=10)$. Nine patients $(30 \%)$ developed CPM and EPM simultaneously. Topographic distribution of extrapontine lesions whether alone or in combination with CPM were as follows: white matter (centrum semiovale), $41.4 \%(n=12)$; corpus callosum, $41.4 \%(n=12)$; basal ganglia, $34.5 \%(n=10)$; hippocampus, $34.5 \%(n=10)$; cerebellum, $34.5 \%(n=10)$; and cortex, $10.3 \%(n=3)$. When we examined the topographic distribution of ODS lesions, 37.9\% $(n=11)$ had both supra- and infratentorial structure involvement, while 20.7\% $(n=6)$ had supratentorial involvement only and $20.7 \%(n=6)$ had infratentorial involvement alone.

\section{Vascular Changes}

Seven patients $(23.3 \%)$ had vascular events, including infarction $20 \%(n=6)$ and subdural hematoma and sinus venous thrombosis $(n=1)$.

\section{Brain and CSF Volume Changes}

Seven patients $(23.3 \%)$ had changes in cerebral volume that manifested as cerebral edema in $16.7 \%(n=5)$ and shrinkage in $6.7 \%$ $(n=2)$. Two patients had changes in CSF volume $(6.7 \%)$ in the form of hydrocephalus $(n=1)$ and subdural effusion $(n=1)$. After correction of $\mathrm{Na}+, 1$ patient had reversibility of brain-volume shrinkage. ${ }^{13}$

\section{Clinical Outcome and Follow-Up}

The clinical outcome after acute hypernatremic challenge was variable. Among reported cases, 8 patients achieved satisfactory recovery $(26.7 \%)$ with minimal neurologic deficits. On the other hand, 17 patients sustained persistent neurologic deficits with significant morbidities (56.7\%), while 5 (16.7\%) died between 12 hours and 3 weeks after presentation. Eight patients had follow-up MR imaging between 2 weeks and 10 months after the onset of osmotic insult. Radiologic findings were variable. Complete neuroradiologic recovery was documented in 3 patients. ${ }^{12-14}$ Residual findings in the form of brain atrophy, ${ }^{23} \mathrm{de}-$ crease in ODS-related T2 signal intensities, ${ }^{26,27,30}$ and patchy contrast enhancement ${ }^{23}$ were reported.

\section{Correlations among Variables}

Younger age was associated with white matter $(P=.05, t$ test $)$, corpus callosum $(P=.010, t$ test $)$, and basal ganglia $(P=.007$, $t$ test) lesions but not with CPM, other EPM sites, or vascular lesions. However, older age was associated with brain volume changes, in the form of edema and shrinkage, compared with younger age $(P=.035, t$ test $)$.
Clinical symptoms on presentation (altered mental status, GCS score, seizures, cranial nerve dysfunction, and motor deficit) had no association with radiologic patterns. The presence of seizures was not associated with development of edema, and development of edema was not related to initial $\mathrm{Na}^{+}$level, serum osmolality, or correction rate. GCS and initial $\mathrm{Na}^{+}$level were not associated with the severity of outcome $(P>.05$, linear regression).

The mean demyelination score was $2.6 \pm 2$, indicating that a number of pontine and extrapontine areas were affected by demyelination. The spatial distribution of demyelinating lesions was not the same across categories of comorbidities ( $P=.01$, MannWhitney). The presence of comorbidities was significantly associated with EPM lesions, namely corpus callosum $(P=.003$, linear regression), WM $(P=.039$, linear regression $), \mathrm{BG}(P=.024$, linear regression $)$, and hippocampus $(P=.024$, linear regression). Among patients with ODS, those with comorbidities had lower $\mathrm{Na}^{+}$levels, while the presence of indirect CNS pathology (hepatic or renal failure) predisposed to development of ODS at lower levels of $\mathrm{Na}^{+}$. The clinical profile, symptoms, and $\mathrm{Na}^{+}$ levels did not predict the development of demyelinating lesions on MR imaging $(P>.05$, linear regression). The presence of vascular complications was not predicted by $\mathrm{Na}^{+}$level, osmolality, and correction rate, and it was not related to age, comorbid history, etiology, or outcome $(P>.05$, linear regression).

Initial $\mathrm{Na}^{+}$level correlated with hippocampus involvement $(P=.003$, linear regression) but not with other extrapontine lesions (WM, corpus callosum, BG, cerebellum, and cortex). $\mathrm{Na}^{+}$ correction rate was not associated with a pattern of radiologic changes or severity of outcome $(P>.05$, linear-by-linear association).

In general, older age was significantly associated with better recovery $(P=.036$, linear regression). The presence of ODS lesions was associated with persistent neurologic dysfunction $(P=$ .027 , Pearson $\chi^{2}$ ), while the presence of EPM lesions reduced the probability of full recovery (odds ratio $=0.118 ; 95 \%$ confidence interval, $0.017-0.802 ; P=.029$, binary logistics).

Poor outcome, defined by the presence of long-term neurologic deficits or death, was found to be significantly associated with higher lesion load ( $P=.021$, linear-by-linear association). Most interesting, among patients with WM $(P=.009$, Fisher exact test), BG ( $P=.027$, Fisher exact test $)$, and hippocampal lesions $(P=.027$, Fisher exact test), none had achieved satisfactory recovery. These results suggest that the absence of these lesions on MR imaging was in favor of better recovery. Conversely, vascular complications and parenchymal volume changes were not associated with the severity of outcome $(P>.05$, linear regression).

\section{DISCUSSION}

In this review, to better delineate the impact of hypernatremic challenge on the CNS, we analyzed the clinical and neuroradiologic profiles of 30 previously reported cases. The results show that altered mental status was the most commonly reported symptom and ODS was the prevailing radiologic pattern. In the following sections, we discuss the spectrum of clinical and radiologic manifestations and their association with other factors in- 
cluding age, presence of pre- and comorbid conditions, the absolute level of $\mathrm{Na}^{+}$, the magnitude of $\mathrm{Na}^{+}$change from baseline, the duration of hypernatremic insult, and the potential effects of $\mathrm{Na}^{+}$ correction rate.

\section{Clinical Characteristics of Patients with Hypernatremic Challenge}

The clinical symptoms of CNS injury ranged from subtle changes of cognitive functions to life-threating autonomic and brain stem dysfunction and death. This spectrum of clinical manifestations is similar to that found in hyponatremia. ${ }^{31,32}$

In our review, clinical symptoms did not correlate with age, presence of comorbid conditions, initial $\mathrm{Na}^{+}$, serum osmolality, or $\mathrm{Na}^{+}$correction rate. This finding would suggest that neither type of clinical symptom nor its severity is influenced by the severity of osmotic challenge. However, in hyponatremia, some studies reported no association between the degree of osmotic derangement and the clinical situation, ${ }^{33,34}$ while some reported that lower $\mathrm{Na}^{+}$level and more rapid $\mathrm{Na}^{+}$correction rates ${ }^{35}$ were associated with a more severe clinical presentation. It appears that the absolute level of $\mathrm{Na}^{+}$, whether abnormally high or low, does not result in a specific set of symptoms and that the emerging clinical presentation is shaped by different sets of factors and mechanisms impacting various components of the neuronal apparatus, including neurons, glia, blood-brain barrier, and CSF. The presence of additional metabolic derangements like hypokalemia $^{36}$ and hyperglycemia ${ }^{2}$ is considered an independent risk factor for ODS development, which is not covered in this review.

All patients included in this review endured acute hypernatremia. While cases of chronic hypernatremia were not included, the distinction between acute and chronic challenge is important because different mechanisms of brain-volume regulation exhibit differential dynamics in acute-versus-chronic osmotic challenge. Data emerging from experimental animal studies indicate that under extreme osmotic challenge, the engagement of organic brain osmoles occurs later in the course of osmotic insult and develops more slowly than the instant changes in inorganic ions, potentially setting a course of different changes in brain tissue. In acute hypernatremia, osmotic differences are actually subtle due to rapid ionic and water shifts across the cell membrane with little effect on cell volume but with significant effect on extracellular space volume, which decreases significantly. In chronic hypernatremia, organic osmoles seem to play a much more important role in helping to maintain the osmotic gradient. ${ }^{37-40}$ The temporal dissociation between these 2 adaptive osmoregulating mechanisms, whether at the acute or chronic stage, could account, at least in part, for the nature and severity of neurologic symptoms. General clinical observations suggest that patients with acute hypernatremia or hyponatremia develop a more clinically severe condition and have worse outcomes that those with chronic challenges. $^{41}$

\section{Neuroradiologic Features of Patients with Hypernatremic Challenge}

Examination in our study of neuroradiologic features of hypernatremic challenge showed the prevailing appearance of ODS (CPM or EPM or both) followed by cerebral volume changes (edema and shrinkage) and vascular complications (hemorrhage and infarction).

\section{Osmotic Demyelination Syndrome}

CPM and EPM (ODS) are classically described as clinical and radiologic manifestations of osmotic myelinolysis, affecting different brain regions, confined to the pons in cases of CPM and extrapontine sites in cases of EPM. The radiologic description of CPM entails the presence of "trident-shaped" symmetric hypointense lesions on $\mathrm{T} 1$ and corresponding hyperintense lesions on T2-weighted images located in the brain stem, and sparing of other brain regions. ${ }^{42}$ In cases of EPM, the same features are noted typically in sites such as the internal capsule, basal ganglia, cerebellum, and cerebrum. ${ }^{43}$

The neuroradiologic features of ODS lesions in the context of hyponatremia are frequently reported not to match the severity of clinical symptoms because the features might be absent despite clinical abnormalities or at times may persist well beyond clinical resolution. ${ }^{44,45}$ We observed similar clinical-radiologic dissociation in cases of hypernatremia. This may potentially be explained by the variability of time intervals between the onset of symptoms and radiologic imaging, the sensitivity of the radiologic technique used, or the presence of gliosis-related abnormal signals that persists well beyond clinical recovery. ${ }^{46}$

In reviewed data, other variables seemed to be more correlated. The presence of an indirect CNS comorbid condition (hepatic or renal failure) before hypernatremic challenge rendered the brain more vulnerable to ODS even with mildly elevated $\mathrm{Na}^{+}$ levels in comparison with patients with no comorbid conditions. More specifically, those patients presented with more corpus callosum, WM, BG, and hippocampus lesions. Patients with liver disease or those who had transplantation are more at risk for developing CNS complications, including ODS, probably secondary to metabolic and cerebral hemodynamic derangements ${ }^{47,48}$ that might render osmotic regulatory mechanisms insufficient.

Hippocampal lesions were reported in more than one-third of patients and were associated with higher initial $\mathrm{Na}^{+}$levels. The high hippocampal involvement may be related to its high vulnerability to the neurotoxic effects of osmotic derangements ${ }^{49}$ and systemic stresses in general. ${ }^{50,51}$

In reviewed cases, contrast enhancement associated with ODS lesions was reported in 1 patient, ${ }^{23}$ which has also been reported in some cases of hyponatremia. ${ }^{52}$ Although inconsistently found, contrast enhancement is in agreement with the pathophysiologic changes observed in experimental models of ODS, in which BBB disruption occurs secondary to osmotic stress and is thought to be one of the leading factors in pathogenesis of ODS. A disruption of the BBB during the first 24 hours of hyponatremia was found to be associated with a $70 \%$ risk of developing demyelination. ${ }^{53}$ By contrast, the risk of developing subsequent demyelination was only $8 \%$ when the BBB was intact. ${ }^{53}$ Furthermore, the use of dexamethasone, known to protect the $\mathrm{BBB}$, was reported to decrease the risk of ODS both in animal studies ${ }^{54}$ and case reports. ${ }^{55}$

Cortical laminar necrosis has been described occasionally in patients with severe ODS that follows rapid correction of hyponatremia. ${ }^{56-59}$ In the present study, we identified 2 patients pre- 
senting with cortical laminar necrosis. ${ }^{23,24}$ These lesions may be associated with hypoxia-ischemia, which may coexist with ODS, or severe demyelination that subsequently triggers cortical neuronal loss. It has been suggested that cortical lesions in the context of ODS present more often with ischemia than with demyelination. ${ }^{60}$

\section{Topographic Distribution of ODS in Hypernatremia: Relation to Region-Specific Vulnerability}

In ODS, regardless of the etiology, CPM is consistently reported to be more common than EPM or CPM/EPM combined. ${ }^{45,61}$ However, in a large case series of patients with hyponatremia and ODS, the combined form was more common than EPM and CPM alone. ${ }^{33}$ As we have already stated, the reviewed data of hypernatremic osmotic challenge have revealed that EPM was more common than CPM or CPM/EPM combined. Whether this hints at a possible predilection of a hypernatremic challenge for extrapontine sites warrants further investigations.

Age appears to be an important factor in the topographic vulnerability to hyperosmotic conditions. Younger adults had different topographic distributions than older adults, with the white matter, corpus callosum, and basal ganglia particularly affected.

Unlike in the adult population, hypernatremic osmotic brain injury is far more common in the pediatric population. Most interesting, the neurologic manifestations of hypernatremia in the pediatric population are strikingly similar to observations made in adults, with some variation in severity and reversibility. Both $\mathrm{CPM}^{62,63}$ and EPM presentations are frequently encountered in pediatric patients, with extrapontine lesions reported to be thalamic, ${ }^{64,65}$ in the basal ganglia, ${ }^{66}$ and hippocampal. ${ }^{67} \mathrm{De}$ spite similar distributions of lesions, pediatric patients have better recovery and the associated MR imaging lesions are more reversible, ${ }^{41,68}$ results that may be related to age-associated changes in osmotic regulatory mechanisms. It has been shown that water, electrolytes, and organic osmole (mainly taurine) content in the brain of normonatremic rats decreases with age, implying that young brains might have a greater capacity to counteract osmotic perturbations. $^{69}$

Furthermore, experimental data support the notion that different brain regions exhibit different "buffering capacities" to osmolar stress, a feature depending largely on the individual composition of electrolytes and osmolytes. Sodium and chloride ions were found to be highest in the medulla oblongata, while the lowest values were found in the cerebellum and vice versa for potassium. ${ }^{70}$ Organic osmoles were also found to significantly differ among different gray and white matter regions. More specifically, the cortex and cerebellum had the highest concentration of glutamate, glutamine, taurine, and betaine, whereas glycine was predominantly present in the pons and medulla. However, other organic osmoles, such as myo-inositol, creatine, glycerylphosphorylcholine, and glycerylphosphorylethanolamine, were found to be distributed evenly across all brain regions. ${ }^{71}$

Furthermore, different dynamics of changes in ionic and organic osmoles may also contribute to different susceptibility of different brain regions to osmotic challenge. Animal studies show upregulation of $\mathrm{Na}$ /myo-inositol co-transporters in the early stages of acute hypernatremia ${ }^{72}$ and an absence of changes in the concentration of brain organic osmoles. Most interesting, during the de-adaptation (recovery) phase, all osmoles except myo-inositol seem to return to a normal range. This may suggest that myo-inositol may play a role either in the development of cerebral edema accompanying correction of hypernatremia, ${ }^{71}$ or alternatively, it may play a protective role as suggested by some experimental studies, which demonstrated that it reduces cell loss resulting from hypernatremia. ${ }^{73}$ Furthermore, myo-inositol is involved in the maintenance of brain cell volume during severe hypernatremia but not in hyponatremia. ${ }^{74}$ Thus, re-establishment of organic osmoles in hyponatremia may differ among brain regions, with the pons and midbrain exhibiting the least recovery capacity and the cortex and cerebellum having greater potential to regain lost organic osmoles. ${ }^{75}$ Therefore, it is conceivable that brain response to osmotic challenges is influenced by the inherent region-specific differences in inorganic and organic metabolic makeup, preferential temporal and concentration-dependent involvement of osmolytes, and morphologic and functional characteristics of the adjacent BBB apparatus.

Another interesting difference emerging from these data is the topographic distribution of EPM lesions. In reviewed data, white matter and corpus callosum lesions were the most frequently reported $(41.4 \%)$, while cortex involvement was the least reported (10.3\%). In hyponatremic challenge, the cerebellum was the most frequently reported site, while the caudate was the least reported one. ${ }^{61}$ It would be relevant to explore whether the difference in susceptibility to different osmotic challenges may be related to the differential dynamics of response of various brain regions to osmotic challenge as indicated earlier.

\section{Cerebral Volume Changes}

In reviewed cases, cerebral edema associated with hypernatremic challenge correlated with older age but not with clinical symptoms, rise in the $\mathrm{Na}^{+}$level, correction rate, or severity of outcome. In most studies, conventional CT or MR imaging was used to delineate edema-related pathologies. Only 2 studies also used DWI and ADC mapping. ${ }^{30,76}$ The potential advantage of using DWI and ADC stems from the role of cytotoxic and vasogenic edema in the development of lesions, especially in white matter regions, given its highly compartmentalized structure. Using serial ADC mapping at different time points after the onset of symptoms showed lower ADC values early after the onset of symptoms and a gradual increase to normal or above-normal values thereafter. ${ }^{77}$ This finding corresponded to the emergence of cytotoxic edema (lower ADC) and subsequent vasogenic edema (higher $\mathrm{ADC}$ ) at different time intervals. It appears that coupling of DWI and ADC yields higher specificity for ODS lesions, in which lower ADC values correlate better with ODS. ${ }^{76}$

\section{Vascular Complications}

Although less frequently reported in this cohort of patients, vascular events (infarction and hemorrhage) were not uncommon. Five patients had cerebral infarction, and 1 patient had cerebral hemorrhage. Vascular complications were not significantly associated with age, comorbid history, $\mathrm{Na}^{+}$level, osmolality, and correction rate, nor were they linked to outcome.

It is not clear whether these vascular complications in hyper- 
natremia are osmotically driven. Experimental data suggest that cerebral blood flow may be affected by disruption of the BBB and a concomitant widespread vascular endothelium reaction. Most interesting, changes in CBF may be regionally distributed, varying between cortical and subcortical regions depending on the dynamics of development and correction of hypernatremia, ${ }^{53}$ suggesting a differential endothelium response and sensitivity to osmotic stress. Whether CBF changes might account for cerebral events (infarctions or hemorrhages) seen in patients with hypernatremic osmotic challenge would require further study.

\section{Outcomes of Hypernatremic Osmotic Challenge}

In reviewed cases, a slower $\mathrm{Na}^{+}$correction rate ( $>48$ hours) was not associated with the presence of radiologic changes or outcome. However, the exact correction rate was not provided in most of studies; this omission prevented statistical analysis.

In our review, satisfactory recovery was reported in $26.7 \%$ of patients, persistent neurologic dysfunction, in 56.7\%; and death, in $16.7 \%$. In reviews of ODS in the context of hyponatremia, favorable recovery was seen in one-third ${ }^{33}$ and two-thirds, ${ }^{45}$ while mortality was reported in one-fifth of patients. ${ }^{33,45}$

In our review, the outcome was not correlated with the severity of clinical presentation, $\mathrm{Na}^{+}$level, or correction rate, findings compatible with previous observations in cases of hyponatremia $^{34}$ and ODS of different etiologies. ${ }^{45,78}$ However, we found that younger adults had worse outcomes compared with older adults. Moreover, the GCS score was not associated with the outcome, probably due to under-reporting of actual GCS scores in most studies. In a case series of 25 patients with hyponatremia and ODS, older age, premorbid conditions, and pattern of ODS were not found to be predictive of better outcome. However, higher GCS scores, better scores in functional scales, less severe hyponatremia, and absence of hypokalemia predicted favorable outcome after correction of a hyponatremic state. ${ }^{33}$

Finally, clinical outcome was better predicted by the presence, load, and pattern of ODS lesions among reviewed cases. More specifically, the presence of EPM lesions, mainly WM, BG, and hippocampal lesions (but not CPM) reduced the probability of full recovery. Similar associations between radiologic features and outcome were not observed in ODS in the setting of hyponatremia. ${ }^{33,45,79}$

\section{CONCLUSIONS}

The analysis of reviewed cases shows striking similarities between the impact of hyper- and hyponatremic osmotic challenge on the CNS. However, several differences have also emerged. The primary one seems to be related to the appearance and distribution of CNS lesions. EPM lesions were prominent in hypernatremic osmotic challenge; among them, corpus callosum lesions were the most common. However, although most reviewed patients presented with neuroradiologic abnormalities, negative initial imaging did not exclude ODS, suggesting that serial imaging with different modalities may be warranted for detecting subtle changes associated with early cellular destruction and water-diffusion anomalies. Although the lack of comprehensive clinical data related to the correction rate precludes specific conclusions, the data suggest that adhering to clinical guidelines when correcting hypernatremia may decrease the risk but does not necessarily prevent ODS development. Finally, the data suggest that the absence of EPM lesions, namely in the WM, BG, and hippocampus might hold better prognostic value for outcome. The reviewed results could have been potentially influenced by selection bias of patients introduced in the original studies, as well as other immediately nonapparent factors, necessitating further clinical observations to better understand the pathophysiology, impact, and outcome of hypernatremic osmotic challenge.

Disclosures: Milos Ljubisavljevic_UNRELATED: Stock/Stock Options: Various stocks not related to current submission or pharmaceutical or other relevant industries.

\section{REFERENCES}

1. Adams RD, Victor M, Mancall EL. Central pontine myelinolysis: a hitherto undescribed disease occurring in alcoholic and malnourished patients. AMA Arch Neurol Psychiatry 1959;81:154-72

2. McComb RD, Pfeiffer RF, Casey JH, et al. Lateral pontine and extrapontine myelinolysis associated with hypernatremia and hyperglycemia. Clin Neuropathol 1989;8:284-88

3. Kuwahara $\mathrm{H}$. Central pontine myelinolysis associated with pancreatic diabetes. J Neurol 2012;259:353-54

4. Lou F. A good prognosis of central pontine myelinolysis in a type 2 diabetic patient. Ann Acad Med Singapore 2011;40:384-86

5. Akyol A. Association of central pontine myelinolysis and extrapontine myelinolysis in diabetic patient. Neuro Endocrinol Lett 2007;28:252-54

6. Morales-Buenrostro LE, Tellez-Zenteno JF, Posadas-Calleja JG, et al. Pontine myelinolysis after a hypoglycemic episode [in Spanish]. Rev Invest Clin 2002;54:181-84

7. Rajbhandari SM, Powell T, Davies-Jones GA. Central pontine myelinolysis and ataxia: an unusual manifestation of hypoglycaemia. Diabet Med 1998;15:259-61

8. Kilinç M, Benli US, Can U. Osmotic myelinolysis in a normonatremic patient. Acta Neurol Belg 2002;102:87-89

9. Clark WR. Diffuse demyelinating lesions of the brain after the rapid development of hypernatremia. West J Med 1992;157:571-73

10. Verbalis JG. Brain volume regulation in response to changes in osmolality. Neuroscience 2010;168:862-70

11. Gage TP, Vivian G. Hypernatremia after hypertonic saline irrigation of an hepatic hydatid cyst. Ann Intern Med 1984;101:405

12. Albi A, Baudin F, Matmar M, et al. Severe hypernatremia after hypertonic saline irrigation of hydatid cysts. Anesth Analg 2002;95: 1806-08, table of contents

13. Machino T, Machino T, Yoshizawa T. Brain shrinkage due to acute hypernatremia. Neurology 2006;67:880

14. Go M, Amino A, Shindo K, et al. A case of central pontine myelinolysis and extrapontine myelinolysis during rapid correction of hypernatremia [in Japanese]. Rinsho Shinkeigaku 1994;34:1130-35

15. Wacks I, Oster JR, Roth D, et al. Severe cerebral edema in a patient with anasarca and hypernatremia. Clin Nephrol 1992;37:19-22

16. Konno S, Nakagawa T, Yoshida T, et al. A case report of central pontine myelinolysis associated with serum hyperosmolality after open heart surgery [in Japanese]. Kyobu Geka 1993;46:150-54

17. Dobato JL, Barriga FJ, Pareja JA, et al. Extrapontine myelinolyses caused by iatrogenic hypernatremia following rupture of a hydatid cyst of the liver with an amnesic syndrome as sequela [in Spanish]. Rev Neurol 2000;31:1033-35

18. Chua GC, Chua GC, Sitoh YY, et al. MRI findings in osmotic myelinolysis. Clin Radiol 2002;57:800-06

19. Kamijo Y, Soma K, Hamanaka S, et al. Dural sinus thrombosis with severe hypernatremia developing in a patient on long-term lithium therapy. Clinical Toxicology 2003;41:359-62

20. van der Helm-van Mil AH, van Vugt JP, Lammers GJ, et al. Hypernatremia from a hunger strike as a cause of osmotic myelinolysis. Neurology 2005;64:574-75 
21. Al-Sarraf AJ, Haque M, Pudek M, et al. Central pontine myelinolysis after orthotopic liver transplant: a rare complication. Exp Clin Transplant 2010;8:321-24

22. Lee IW, Su MT, Kuo PL, et al. Gestational diabetes and central pontine myelinolysis with quadriplegia: a case report. J Matern Fetal Neonatal Med 2010;23:728-31

23. Szolics M, Ljubisavljevic M, Samir HA, et al. Extrapontine myelinolysis and cortical laminar necrosis caused by severe hypernatremia following peritoneal lavage for ruptured hydatid cyst of the liver: a case report and review of the literature. The Neuroradiology Journal 2010;1:325-31

24. Cagnin A, Manara R, Piron L, et al. Hypernatremia-induced limbic system damage. Anesthesiology 2011;114:175

25. Odier C, Nguyen DK, Panisset M, et al. Central pontine and extrapontine myelinolysis: from epileptic and other manifestations to cognitive prognosis. J Neurol 2010;257:1176-80

26. Chang L, Harrington DW, Milkotic A, et al. Unusual occurrence of extrapontine myelinolysis associated with acute severe hypernatraemia caused by central diabetes insipidus. Clin Endocrinol (Oxf) 2005;63:233-35

27. Kawahara I, Tokunaga Y, Ishizaka S, et al. Reversible clinical and magnetic resonance imaging of central pontine myelinolysis following surgery for craniopharyngioma: serial magnetic resonance imaging studies. Neurol Med Chir (Tokyo) 2009;49:120-23

28. Vin-Christian K, Arieff AI. Diabetes insipidus, massive polyuria, and hypernatremia leading to permanent brain damage. Am J Med 1993;94:341-42

29. Kendall R, Lafuente J, Hosie KB. Central pontine myelinolysis after an operation. J R Soc Med 1997;90:445-46

30. Naik KR, Saroja AO. Seasonal postpartum hypernatremic encephalopathy with osmotic extrapontine myelinolysis and rhabdomyolysis. J Neurol Sci 2010;291:5-11

31. Riggs JE. Neurologic manifestations of electrolyte disturbances. Neurol Clin 2002;20:227-39, vii

32. Adrogué HJ, Madias NE. Hypernatremia. N Engl J Med 2000;342: 1493-99

33. Kallakatta RN, Radhakrishnan A, Fayaz RK, et al. Clinical and functional outcome and factors predicting prognosis in osmotic demyelination syndrome (central pontine and/or extrapontine myelinolysis) in 25 patients. J Neurol Neurosurg Psychiatry 2011;82: $326-31$

34. Menger $\mathrm{H}$. Outcome of central pontine and extrapontine myelinolysis $(\mathbf{n}=44)$. J Neurol 1999;246:700-05

35. Brunner JE, Redmond JM, Haggar AM, et al. Central pontine myelinolysis and pontine lesions after rapid correction of hyponatremia: a prospective magnetic resonance imaging study. Ann Neurol 1990;27:61-66

36. Davenport C, Liew A, Vic Lau P. Central pontine myelinolysis secondary to hypokalaemic nephrogenic diabetes insipidus. Ann Clin Biochem 2010;47:86-89

37. Strange K. Regulation of solute and water balance and cell volume in the central nervous system. J Am Soc Nephrol 1992;3:12-27

38. De Petris L, Luchetti A, Emma F. Cell volume regulation and transport mechanisms across the blood-brain barrier: implications for the management of hypernatraemic states. Eur J Pediatr 2001;160: 71-77

39. Holliday MA, Kalayci MN, Harrah J, et al. Factors that limit brain volume changes in response to acute and sustained hyper- and hyponatremia. J Clin Invest 1968;47:1916-28

40. Cserr HF, DePasquale M, Nicholson C, et al. Extracellular volume decreases while cell volume is maintained by ion uptake in rat brain during acute hypernatremia. J Physiol 1991;442:277-95

41. Arieff AI. Central nervous system manifestations of disordered sodium metabolism. Clin Endocrinol Metab 1984;13:269-94

42. Rippe DJ, Edwards MK, D'Amour PG, et al. MR imaging of central pontine myelinolysis. J Comput Assist Tomogr 1987;11:724-26

43. Bekiesińska-Figatowska M, Bulski T, Rózyczka I, et al. MR imaging of seven presumed cases of central pontine and extrapontine myelinolysis. Acta Neurobiol Exp (Wars) 2001;61:141-44

44. Clifford DB, Gado MH, Levy BK. Osmotic demyelination syndrome: lack of pathologic and radiologic imaging correlation. Arch Neurol 1989;46:343-47

45. Graff-Radford J, Fugate JE, Kaufmann TJ, et al. Clinical and radiologic correlations of central pontine myelinolysis syndrome. Mayo Clin Proc 2011;86:1063-67

46. Thompson PD, Miller D, Gledhill RF, et al. Magnetic resonance imaging in central pontine myelinolysis. J Neurol Neurosurg Psychiatry 1989;52:675-77

47. Ardizzone G, Arrigo A, Schellino MM, et al. Neurological complications of liver cirrhosis and orthotopic liver transplant. Transplant Proc 2006;38:789-92

48. Ardizzone G, Arrigo A, Panaro F, et al. Cerebral hemodynamic and metabolic changes in patients with fulminant hepatic failure during liver transplantation. Transplant Proc 2004;36:3060-64

49. Goldman MB, Torres IJ, Keedy S, et al. Reduced anterior hippocampal formation volume in hyponatremic schizophrenic patients. Hippocampus 2007;17:554-62

50. Fotuhi M, Do D, Jack C. Modifiable factors that alter the size of the hippocampus with ageing. Nat Rev Neurol 2012;8:189-202

51. Vexler ZS, Ayus JC, Roberts TP, et al. Hypoxic and ischemic hypoxia exacerbate brain injury associated with metabolic encephalopathy in laboratory animals. J Clin Invest 1994;93:256-64

52. Dervisoglu E, Yegenaga I, Anik Y, et al. Diffusion magnetic resonance imaging may provide prognostic information in osmotic demyelination syndrome: report of a case. Acta Radiol 2006;47:208 -12

53. Adler S, Verbalis JG, Meyers S, et al. Changes in cerebral blood flow and distribution associated with acute increases in plasma sodium and osmolality of chronic hyponatremic rats. Exp Neurol 2000;163: 63-71

54. Ke QH, Chen JH, Zheng SS, et al. Prevention of central pontine myelinolysis in rats by early treatment with dexamethasone [in Chinese]. Zhejiang Da Xue Xue Bao Yi Xue Ban 2006;35:424-29

55. Sakamoto E, Hagiwara D, Morishita Y, et al. Complete recovery of central pontine myelinolysis by high dose pulse therapy with methylprednisolone [in Japanese]. Nihon Naika Gakkai Zasshi 2007;96: 2291-93

56. Cho AH, Choi CG, Lee SA. Cortical laminar necrosis associated with osmotic demyelination syndrome. J Clin Neurol 2005;1:174-76

57. Niwa K, Matsushima K, Yamamoto M,et al. Morel's laminar necrosis like findings on MRI in a case of extra-pontine myelinolysis [in Japanese]. Rinsho Shinkeigaku 1991;31:327-30

58. Shoji M, Kimura T, Ota K, et al. Cortical laminar necrosis and central pontine myelinolysis in a patient with Sheehan syndrome and severe hyponatremia. Intern Med 1996;35:427-31

59. Susa SD. Acute intermittent porphyria with central pontine myelinolysis and cortical laminar necrosis. Neuroradiology 1999;41: 835-39

60. Cho AH, Choi CG, Lee SA. Cortical laminar necrosis associated with osmotic demyelination syndrome. J Clin Neurol 2005;1:174-76

61. Martin RJ. Central pontine and extrapontine myelinolysis: the osmotic demyelination syndromes. J Neurol Neurosurg Psychiatry 2004;75(suppl 3):iii22-28

62. Shah B, Tobias JD. Osmotic demyelination and hypertonic dehydration in a 9-year-old girl: changes in cerebrospinal fluid myelin basic protein. J Intensive Care Med 2006;21:372-76

63. Mastrangelo S, Arlotta A, Cefalo MG, et al. Central pontine and extrapontine myelinolysis in a pediatric patient following rapid correction of hypernatremia. Neuropediatrics 2009;40:144-47

64. Ozdemir H, Kabakus N, Kurt AN, et al. Bilateral symmetrical hypodensities in the thalamus in a child with severe hypernatraemia. Pediatr Radiol 2005;35:449-50

65. Achiwa S, Ando K, Ishikura R, et al. A case of extrapontine myelinolysis precipitated by correction of a hyper-osmolar state [in Japanese]. Nihon Igaku Hoshasen Gakkai Zasshi 2004;64:310-12

66. AlOrainy IA, O'Gorman AM, Decell MK. Cerebral bleeding, in- 
farcts, and presumed extrapontine myelinolysis in hypernatraemic dehydration. Neuroradiology 1999;41:144-46

67. Brown WD, Caruso JM. Extrapontine myelinolysis with involvement of the hippocampus in three children with severe hypernatremia. J Child Neurol 1999;14:428-33

68. Fahrbach J, Rozzelle CJ. Transient ventriculomegaly in a child presenting with hypernatremia: case report. J Neurosurg 2006;104 (3 suppl):192-94

69. Trachtman H, Yancey PH, Gullans SR. Cerebral cell volume regulation during hypernatremia in developing rats. Brain Res 1995;693: $155-62$

70. Reulen HJ, Hase U, Fenske A, et al. Extracellular space and electrolyte distribution in grey and white matter of the dog brain [in German]. Acta Neurochir (Wien) 1970;22:305-25

71. Lien YH, Shapiro JI, Chan L, et al. Effects of hypernatremia on organic brain osmoles. J Clin Invest 1990;85:1427-35

72. Minami $Y$, Inoue K, Shimada S, et al. Rapid and transient up-regulation of $\mathrm{Na}+$ /myo-inositol cotransporter transcription in the brain of acute hypernatremic rats. Brain Res Mol Brain Res 1996;40:64-70

73. Hijab S, Havalad S, Snyder AK. The role of organic osmolytes in the response of cultured astrocytes to hyperosmolarity. Am J Ther 2011;18:366-70

74. Trachtman $\mathrm{H}$. Cell volume regulation: a review of cerebral adaptive mechanisms and implications for clinical treatment of osmolal disturbances. I. Pediatr Nephrol 1991;5:743-50

75. Lien YH. Role of organic osmolytes in myelinolysis: a topographic study in rats after rapid correction of hyponatremia. J Clin Invest 1995;95:1579-86

76. Chu K, Kang DW, Ko SB, et al. Diffusion-weighted MR findings of central pontine and extrapontine myelinolysis. Acta Neurol Scand 2001;104:385-88

77. Hurley RA, Filley CM, Taber KH. Central pontine myelinolysis: a metabolic disorder of myelin. I Neuropsychiatry Clin Neurosci 2011;23:369-74

78. Louis G, Megarbane B, Lavoué S, et al. Long-term outcome of patients hospitalized in intensive care units with central or extrapontine myelinolysis. Crit Care Med 2012;40:970-72

79. Menger H, Mackowski J, Jörg J, et al. Pontine and extra-pontine myelinolysis: early diagnostic and prognostic value of cerebral CT and MRI [in German]. Nervenarzt 1998;69:1083-90 